\title{
Los retos de la Educación Física en el Siglo XXI Challenges of Physical Education in XXI Century
}

\author{
Víctor Manuel López Pastor, Dario Pérez Brunicardi, Juan Carlos ManriqueArribas; Roberto Monjas Aguado
}

Universidad de Valladolid(España)

\begin{abstract}
Resumen. Este artículo aporta una serie de reflexiones y consideraciones sobre los retos a los que se tienen que enfrentar la Educación Física (EF) en estas primeras décadas del Siglo XXI. Desde nuestro punto de vista, los principales retos que tiene la EF actualmente en nuestro país son los siguientes: (1) plantearse de forma reflexiva y sistemática qué queremos que aprenda nuestro alumnado en EF; (2) tener razones claras y poderosas sobre lo que puede aportar la EF a la educación integral de las personas; y, (3) superar definitivamente algunos problemas de profesionalidad entre los docentes que venimos reproduciendo durante los últimos 30 años. Realizamos una propuesta concreta para poder avanzar sobre estos retos. Durante las tres últimas décadas han surgido en España muchas propuestas innovadoras en EF que pueden ser muy útiles para ir dando una respuesta educativamente valiosa a estos retos. La mayor parte de dichas propuestas han sido generadas por el profesorado de EF a través de dinámicas de investigación-acción e investigación en el aula. En la mayoría de los casos han sido generadas de forma grupal, en base a grupos colaborativos de maestros, aunque también podemos encontrar algunas individuales. Terminamos el artículo con una primera aproximación a las relaciones que parecen existir entre las competencias profesionales del profesorado de EF y los retos de la EF en la sociedad y la escuela actual.
\end{abstract}

Palabras clave. Educación Física, Retos actuales, Finalidades educativas, Aprendizaje, Competencias Profesionales.

Abstract. This paper seeks to contribute some thoughts and considerations on the challenges that Physical Education (PE) has to face in these early decades of the $21^{\text {st }}$ century. From our point of view, the main challenges that PE must currently face in our country are: (1) considering thoughtfully and systematically what we want our students to learn in PE; (2) have clear and powerful reasons about what PE can bring to the comprehensive education of people; and (3) overcome some problems of professionalism among teachers we have been recreating for the past 30 years. We make a specific proposal to move forward on these challenges. During the past three decades many innovative proposals in PE that can be very useful for giving an educationally valuable response to these challenges have arisen in Spain. Most of these proposals have been generated by PE teachers through dynamic action research and research in the classroom. In most cases they have been generated as a group, based on collaborative groups of teachers, but we can also find some individual proposals. We ended the article with a first approach to relationships that seem to exist between the professional skills of PE teachers and PE challenges in society and in contemporary school.

Keywords. Physical Education, Current Challenges, Educational Purposes; Learning, Professional Skills

\section{Introducción}

Durante las últimas décadas se han ido planteando diferentes retos para conformar un modelo más completo de EF: la adherencia a la actividad física en el tiempo, la condición física orientada a la salud, la recreación, la iniciación deportiva, la educación en valores, el bilingüismo, etc.

Bajo nuestro punto de vista, es un debate banal o, al menos, adulterado. Desde nuestra perspectiva, el principal reto de la EF actual se relaciona con tener claras sus finalidades, sus grandes objetivos, su razón de ser. Quizás podríamos resumirlo con estas preguntas: ¿cuál es la principal finalidad de la EF? y ¿¿cuál el papel que la EF debe jugar en la educación formal?

A lo largo de este texto vamos a intentar realizar una aportación a este debate. Primero plantearemos cuáles son, según nuestros criterios, los principales retos de la EF actual y su relación con las competencias profesionales que debe dominar el profesorado de EF. Posteriormente revisaremos cómo muchas de las propuestas de innovación en EF que han surgido en España en las tres últimas décadas pueden ayudar a ir avanzando en la superación de estos retos y a lograr que la EF cumpla el papel que merece en la educación formal.

Durante la segunda mitad del siglo XX la EF parecía tener una finalidad más o menos clara, su orientación hacia el rendimiento físico y deportivo, debido a la repercusión del deporte en la sociedad. Esto es, la EF se orientaba hacia el entrenamiento de las capacidades físicas y habilidades motrices del alumnado y a una iniciación deportiva muy centrada en aspectos técnicos, basada en el uso de metodologías directivas y analíticas y un enfoque predominantemente dirigido a la competición y al rendimiento deportivo. Por tanto, predominaba lo que Tinning (1996) define como «discursos de EF orientados al rendimiento». Este enfoque de EF todavía sigue vivo en muchos de nuestros centros educativos, especialmente en educación secundaria y en algunos centros de formación inicial del profesorado de EF (FIPEF). Pero a su vez, desde hace ya tres décadas, también han ido surgiendo numerosas corrientes

Fecha recepción: 24-07-15- Fecha envío revisores: 24-07-15- Fecha de aceptación: 18-11-15 Víctor Manuel López Pastor vlopez@mcpc.uva.es críticas ante ese tipo de enfoques y discursos de EF, generándose propuestas que pretenden ser una alternativa a dicha forma de entender $y$ practicar la EF. El razonamiento en el cual se sustentan dichas críticas se basa en que una $\mathrm{EF}$ que se desarrolla dentro de un sistema educativo no puede tener como principal finalidad el mero entrenamiento de los cuerpos o la detección, selección y entrenamiento de posibles talentos deportivos. Frente a «los discursos orientados al rendimiento», Tinning (1996) defiende los «discursos orientados a la participación», en los que lo realmente importante es que toda la población realice actividad física regularmente, tenga interés por ella y logre, con ello, el bienestar, independientemente de sus capacidades físicas y deportivas, como corresponde a una materia que forma parte de un sistema educativo universal y obligatorio. De este modo, se relega el interés por el rendimiento al ámbito del deporte federado, algo lícito fuera de la escuela. Entendemos que el acento debe ponerse en la importancia de que la EF contribuya a la formación integral del alumnado. Por tanto, debe tener una orientación formativa.

Las respuestas a las preguntas: ¿cuál es entonces la finalidad que debe cumplir laEF? y ¿cuál el papel que la EF debe jugar en la educación formal?, ya parecen intuirse.

Nuestra perspectiva sobre los principales retos de la educación física en la actualidad

Desde nuestro modo de pensar, los principales retos a los que sigue enfrentándose la EF en España en este principio de siglo son tres: (a) plantearse de forma reflexiva y sistemática qué queremos que aprenda nuestro alumnado en EF; (b) tener razones claras y poderosas sobre lo que puede aportar la EF a la educación integral de las personas; y (c) lograr definitivamente la profesionalidad que venimos demandando durante los últimos 30 años.

Respecto al primero, llevamos 20 años de profesión docente observando con preocupación que una parte del profesorado de EF no tiene un planteamiento claro y estructurado sobre qué quiere que aprenda su alumnado en sus clases. También comprobamos que otra parte limita sus ambiciones a lo físico y motriz y a que se lo pasen bien sus educandos. En Primaria sigue siendo frecuente la corriente que ha convertido las clases de EF en un simple recopilatorio de juegos motores. Da igual cuál es el contenido a trabajar o la organización metodológica a emplear, la estructura de sesión siempre es la misma. Esta estructura de clase atien- 
de al famoso eslogan: «entretenido, feliz y cansado». Desde este planteamiento, un buen profesor de EF es el que conoce un buen repertorio de juegos y sabe combinarlos adecuadamente a lo largo del curso. En Secundaria este mismo planteamiento también se está extendiendo notablemente combinando situaciones lúdicas con prácticas más propias del entrenamiento y rendimiento deportivo, sin que gran parte del profesorado sea capaz de superarlas. Por ejemplo, la aplicación de test de condición física cuando llega la hora de la calificación o el empleo de una enseñanza analítica y excesivamente técnica de los deportes.

Respecto al segundo, los efectos de la actividad física sobre la población escolar están suficientemente documentados. Estos se centran tanto en aspectos de mejora fisiológica, como psicológica o social. Por ejemplo, la actividad física ayuda a reducir la grasa corporal, reduce los niveles de triglicéridos, reduce la presión sanguínea, mejora la fuerza y la resistencia muscular, posee efectos positivos sobre los síntomas de ansiedad y depresión, sobre el autoconcepto físico, la concentración, la memoria, el comportamiento en clase, las relaciones con los compañeros y, algunos datos sugieren, un incremento relativo del rendimiento académico (Programa Perseo, 2007, 24).

El planteamiento de una EF que facilite la consecución de los efectos anteriormente citados debe provocar un efecto positivo en el aumento de la actividad física precisamente fuera del contexto escolar. La aplicación, por tanto, de unos estilos de enseñanza basados en la promoción de la salud preparan mejor el camino para que el alumnado mantenga un modo de vida físicamente activo a largo plazo (Devís y Peiró, 1993). Por ello, también habrá que involucrar a la familia y a la comunidad, puesto que el tiempo de práctica durante las clases de EF es claramente insuficiente para conseguir estos objetivos. Estas clases deben ser, por tanto, promotoras de actividad física y deportiva, de juegos y de hábitos saludables. El nuevo currículo de EF en Primaria (RD 126/ 2014) está impregnado de contenidos relacionados con la salud (biológica, social y psicológica), aunque predominan los de corte físico. Se pretende así hacer consciente al alumnado de las consecuencias de la vida sedentaria (Pastor-Vicedo, Gil-Madrona, Prieto-Ayuso y GonzálezVillora 2015, 139).

Respecto al tercer reto, en López et al. $(2003,2004,2005)$ puede encontrarse una recopilación y explicación detallada de los más importantes: (a) evaluación-calificación por test de condición física (CF) y habilidad; (b) la tradición del «juego libre» 0 «dejar balones»; (c) la falta de programación y secuenciación clara y organizada a lo largo de cada etapa educativa; (d) copiar los malos hábitos de otras materias (dictado de apuntes, exámenes teóricos, elevados porcentajes de suspensos, etc.); (e) la EF como simple recopilación de juegos motores que se van mezclando; (f) la reproducción de estructuras de sesión orientadas al mero entrenamiento físico o al entretenimiento; o (g) la escasa formación permanente, en sus diferentes modalidades (cursos, grupos de trabajo, proyectos formativos, lectura, asistencia a congresos profesionales, etc.).

Es cierto que poco a poco un porcentaje cada vez mayor del profesorado de EF está alcanzando estos retos, pero a veces estos procesos de cambio se van extendiendo con lentitud. Lo hemos percibido al asistir de forma habitual a jornadas y congresos profesionales, al hablar con colegas de otras ciudades y provincias durante los cursos en diferentes lugares de nuestro país, al leer artículos y libros que se publican en revistas profesionales y científicas, o cuando observamos las prácticas educativas en los entornos cercanos. A veces se ve el vaso medio vacío, se observa que el modus operandi en esta profesión sigue reproduciéndose curso tras curso. Aunque también, otras muchas veces podemos disfrutar viendo el vaso medio lleno, al observar cómo cada vez hay más compañeras y compañeros implicados en procesos de innovación, cambio y mejora, intentando aportar su granito de arena para ir consiguiendo una EF de mayor valor educativo y que desarrolle todo el potencial que encierra. Por ejemplo, pueden encontrarse interesantes relatos sobre los procesos de desarrollo profesional de profesores de EF en Barba(2006), Córdoba (2015) y González-Calvo y Barba (2013), que demuestran que la implicación en procesos de formación permanente y de reflexiónacción guarda una fuerterelación con la mejora de la práctica profesional.
Córdoba et al. (2016) muestran un interesante relato de escritura colectiva sobre cómo la participación en un grupo de trabajo afecta positivamente al desarrollo profesional de los docentes que forman parte del mismo y a la calidad de su práctica educativa. Similares resultados pueden encontrarse también en el estudio de Pedraza y López (2015) sobre la influencia que tiene formar parte de un grupo de trabajo en la evolución profesional de los maestros de EF que trabajan en la escuela rural, habitualmente como itinerantes. Por su parte, en Pérez-Pueyo (2016) se analiza la evolución de la propuesta denominada «EF por estilo actitudinal», que combina procesos de desarrollo profesional docente individuales y colectivos. En esos casos, los optimistas vuelven a casa más contentos, con cierta «envidia sana» (Pérez-Brunicardi, 2002) por lo que otros colegas logran.

Intentando resolver los retos: ¿Cuáles son las finalidades de la educación física actualmente?

Nosotros entendemos que la EF actual debería tener tres grandes finalidades como área curricular específica dentro de un sistema educativo universal, obligatorio y público:

(1) el desarrollo físico-motriz del alumnado;

(2) la creación y recreación de la cultura física del alumnado; y

(3) su aportación al planteamiento global de desarrollo integral del alumnado, como ciudadanos de una sociedad democrática.

Las dos primeras finalidades son específicas y propias de nuestra área. Si nosotros no nos encargamos de ellas, nadie más lo va a hacer. La tercera es una finalidad global, que nos identifica, relaciona y une con el resto de compañeros de otras áreas, con todos los profesionales de la educación y con toda la comunidad educativa.

En esta tercera finalidad podemos realizar dos aportaciones fundamentales: (a) el desarrollo, en mayor o menor medida, de las competencias clave (o básicas); y (b) una importante aportación al desarrollo integral de nuestro alumnado, desde, con y a través de la motricidad.

Tal como hemos comentado en el apartado anterior, hay muchas propuestas prácticas de EF en primaria y secundaria que no inciden de forma suficiente en las tres finalidades. La mayoría se preocupan exclusivamente de la primera, el desarrollo físico-motriz del alumnado. Pero también pueden encontrarse otras que se centran tanto en la tercera finalidad que se olvidan de lo propio y específico de nuestra área.

Por eso entendemos que es fundamental trabajar de forma global en las tres finalidades, sin olvidar ninguna de ellas. Ahora bien, para conseguir un sistema de aprendizaje claro y coherente, propio y específico del área, es la creación y recreación de la cultura física del alumnado la que debe servirnos de guía. En este sentido, la propuesta más completa y adecuada que conocemos es la programación en EF por Dominios de Acción Motriz (DAM). Esta permite organizar y secuenciar mejor los aprendizajes a desarrollar por el alumnado de EF a lo largo de su paso por el sistema educativo. En este sentido, un desarrollo curricular completo para el área de EF en Primaria fue elaborado por Larraz (2002, 2004) para Aragón. Hace poco más de un año el Ministerio de Educación ha aprobado una nueva propuesta curricular oficial que está basada en gran medida en los DAM como bloques de contenidos (MECD, 2014). En dicho Real Decreto se afirma que la Finalidad principal de la EF es desarrollar en las personas su competencia motriz, entendida como la integración de: (a) los conocimientos; (b) los procedimientos; (c) las actitudes; y (d) los sentimientos, vinculados fundamentalmente a la conducta motora.

Durante las tres últimas décadas han surgido en España muchas propuestas innovadoras en $\mathrm{EF}$ que pueden ser muy útiles para ir dando una respuesta educativamente valiosa a estos retos. La mayor parte de dichas propuestas han sido generadas por el profesorado de EF a través de dinámicas de investigación-acción e investigación en el aula. En la mayoría de los casos han sido generadas de forma grupal, en base a grupos colaborativos de maestros, aunque también podemos encontrar algunas individuales. En López-Pastor y Gea (2010) se realiza una revisión de dichas propuestas, agrupándolas en cinco grandes corrientes o tendencias: (1) el salto hacia los discursos de participación y los planteamientos comprensivos en EF; (2) propuestas de aprendizaje 
inducido en EF (el aprendizaje a través de la manipulación de espacios y materiales); (3) propuestas que se apoyan en, o trabajan de modo explícito, la Educación en Valores y las actividades físicas cooperativas en EF; (4) propuestas de EF crítica; (5) propuestas basada en la programación por dominios de acción motriz en EF. En la tabla 1 realizamos un resumen esquemático de las mismas.

\begin{tabular}{|c|c|}
\hline Tendencia o corriente & Principales propuestas de cada tendencia o corriente \\
\hline $\begin{array}{l}\text { A-El salto hacia los } \\
\text { discursos de } \\
\text { participación y los } \\
\text { planteamientos } \\
\text { comprensivos en EF. }\end{array}$ & $\begin{array}{l}\text {-EF para la salud; los juegos modificados en EF y el enfoque } \\
\text { comprensivo en iniciación deportiva } \\
\text {-La investigación-acción en EF y el trabajo colaborativo en la } \\
\text { formación permanente del profesorado } \\
\text {-Metodología por Proyectos en EF } \\
\text {-Los temas transversales y la educación en valores en EF } \\
\text {-Atención a la diversidad en EF y EF en contextos específicos } \\
\text {-Alternativas en EF, utilización de material de desecho,... }\end{array}$ \\
\hline $\begin{array}{l}\text { B-Propuestas de } \\
\text { aprendizaje inducido } \\
\text { en EF; el aprendizaje a } \\
\text { través de la utilización } \\
\text { de espacios, materiales }\end{array}$ & $\begin{array}{l}\text {-Los Espacios de “Acción-Aventura } \\
\text {-Los ambientes de aprendizaje } \\
\text {-El tratamiento pedagógico de lo corporal } \\
\text {-Educación Psicomotriz y Educación por el movimiento } \\
\text {-Espacios de aventura y fantasía }\end{array}$ \\
\hline $\begin{array}{l}\text { C-Propuestas de } \\
\text { innovación que se } \\
\text { apoyan en, o trabajan } \\
\text { de modo explícito la, } \\
\text { Educación en Valores } \\
\text { y las actividades } \\
\text { físicas cooperativas en } \\
\text { Educación Física. } \\
\end{array}$ & $\begin{array}{l}\text {-La educación en valores en EF } \\
\text {-La utilización de actividades físicas cooperativas en EF. } \\
\text {-El aprendizaje cooperativo en EF } \\
\text {-La EF para la paz, la convivencia y la Integración; } \\
\text {-La EF basada en actitudes; } \\
\text {-La propuesta "valors en joc": } \\
\text {-Los programas de responsabilidad personal y social en EF. }\end{array}$ \\
\hline $\begin{array}{l}\text { D-Propuestas de } \\
\text { Educación Física } \\
\text { Crítica }\end{array}$ & $\begin{array}{l}\text {-EF Alternativa } \\
\text {-Investigación-acción en EF desde un paradigma crítico } \\
\text {-Propuestas y experiencias de EF crítica } \\
\text {-La autonomía y la autogestión en EF }\end{array}$ \\
\hline $\begin{array}{l}\text { E-Programar por } \\
\text { "Dominios de Acción } \\
\text { Motriz" (DAM) en EF }\end{array}$ & $\begin{array}{l}\text {-Currículum por Dominios de Acción (Praxiología Motriz aplicada al } \\
\text { aula en Primaria) } \\
\text {-Elaboración de Material Curricular en EF y una programación de EF } \\
\text { en Primaria basada en Dominios de Acción y metodología por } \\
\text { Proyectos en EF. } \\
\text {-Otras propuestas con aplicaciones prácticas basadas en la Praxiología } \\
\text {-Evaluación del alumno en EF desde la Praxiología Motriz }\end{array}$ \\
\hline
\end{tabular}

Por otra parte, a lo largo de los dos números de este monográfico sobre Didáctica de la Educación Física, pueden encontrarse varios artículos que corresponden de forma clara a varias de estas líneas de innovación educativa. En la primera podrían integrarse los trabajos deAlonso, Gómez-Alonso, Pérez-Pueyo y Gutiérrez-García (2016), Castejón (2015), Córdoba et al (2016); Delgado (2015), Flores, Prat y Soler (2015), Gallardo-Fuentes y Carter (2016), González (2016), Monjas, Ponce y Gea (2015) y Romero-Martín, Asún y Chivite (2016). En la segunda el trabajo de Martínez (2016) y Tabernero, Aliseda y Daniel (2016). En la tercera podrían integrarse los artículos de Córdoba (2015), Fernández-Rio y Méndez (2016), Monzonís y Capllonch (2015), Pérez-Pueyo (2016), Ruiz-Omeñaca, Ponce, Sanz y Valdemoros (2015), Velázquez (2015). En la cuarta los trabajos de Martos, Tamarit y Torrent (2016) y Moreno y Poblete (2015). Por último, en la quinta, se podrían incluir los artículos de Julián, Abarca-Sos, Zaragoza \& Aibar (2016) y López et al. (2016).

En el siguiente apartado entramos a fondo en las ventajas y posibilidades que tiene estaúltima línea de innovación einvestigación en la EF: la programación por DAM.

Programar por Dominios de Acción Motriz y el actual currículo oficial en Educación Física

El actual decreto de currículum oficial para el área de EF en primaria (MECD, 2014) basa su organización de «bloques de contenidos» en la programación por DAM. Se trata de propuestas basadas en los planteamientos de la Praxiología Motriz y su aplicación a la EF. Hasta el momento existen pocas propuestas de intervención didáctica publicadas en torno a este planteamiento. Desde nuestro punto de vista, la propuesta más completa y elaborada en estos momentos es la desarrollada por Larraz (2002, 2004, 2009), que ha estado más de quince años organizando toda su programación de EF en Primaria basada en este planteamiento y que diseñó un Currículum para el área de EF en Primaria en base a este planteamiento (Larraz, 2004). Larraz considera que estos dominios organizan las seis grandes clases de experiencias corporales que atraviesan la diversidad de prácticas motrices de la EF escolar, mediante el trabajo de los seis tipos de problemas motores diferentes a los que puede enfrentarse el alumnado y las diferentes relaciones que pueden darse entre la persona y el entorno físico y humano. Cada uno de los DAM tiene rasgos comunes de lógica interna y responde a un tipo de problema motor. Por tanto, las prácticas motrices de cada dominio pueden considerarse homogéneas. En base a esta realidad, es posible agrupar todos los contenidos de EF en torno a estos 6 DAM y organizar y revisar así las programaciones. El propio MECD (2014) especifica esta cuestión, afirmando que la lógica interna de las situaciones o actividades motrices se convierte así en una herramienta imprescindible para la programación en EF.

En la Tabla 2 se realiza una comparativa entre la propuesta de Larraz (2002, 2004, 2009) y el actual currículo oficial de EF en Primaria (LOMCE, 2014). Como puede comprobarse, la organización de bloques de contenido del actual currículo está basada de forma clara en la propuesta de Larraz, con la única diferencia de haber agrupado los dominios de acción 3 y 4 en uno sólo. A pesar de ello, también aparecen problemas de incoherencia interna en los apartados posteriores de criterios de evaluación y estándares de aprendizaje. Desgraciadamente, en el desarrollo posterior que han realizado algunas comunidades autónomas se siguen reflejando graves incongruencias con la propuesta original, como la que presenta la Orden de Castilla y León (Orden EDU/519/ 2014), en la que se demuestra que no se ha entendido la lógica interna de la propuesta oficial y que se siguen reproduciendo los mismos planteamientos tradicionales de los últimos 25 años, en lo que respecta a la organización de bloques de contenidos.

\begin{tabular}{|c|c|}
\hline Denominación DAM Larraz & \begin{tabular}{|l} 
Denominación DAM \\
LOMCE (BOE, \\
01/03/2014)
\end{tabular} \\
\hline $\begin{array}{l}\text { 1- Acciones en un entorno físico estable y sin interacción } \\
\text { directa con otros. Actividades realizadas principalmente de } \\
\text { forma individual. Suelen ser acciones medibles, con parámetros } \\
\text { espaciales o temporales. }\end{array}$ & $\begin{array}{l}\text { 1. Acciones motrices } \\
\text { individuales en entornos } \\
\text { estables. }\end{array}$ \\
\hline $\begin{array}{l}\text { 2-Acciones de oposición interindividual. Actividades en } \\
\text { situaciones de enfrentamiento uno contra uno. }\end{array}$ & $\begin{array}{l}\text { 2. Acciones motrices en } \\
\text { situaciones de oposición. }\end{array}$ \\
\hline $\begin{array}{l}\text { 3-Acciones de cooperación. Hay que resolver un problema } \\
\text { común que requiere la cooperación de todo el grupo. No hay } \\
\text { enfrentamiento con otros grupos ni personas. Todos ganan. } \\
\text { Implica procesos de diálogo, ayuda y solidaridad. }\end{array}$ & $\begin{array}{l}\text { - 3. Acciones motrices en } \\
\text { situaciones de } \\
\text { cooperación, con o sin }\end{array}$ \\
\hline $\begin{array}{l}\text { 4-Acciones de cooperación (**) y oposición. Son acciones } \\
\text { colectivas que requieren la colaboración con las personas del } \\
\text { mismo grupo para superar a las del grupo contrario. Suelen ser } \\
\text { situaciones con alto grado de codificación, en las que cobra } \\
\text { gran importancia el respeto de unas normas y reglas de juego. }\end{array}$ & $\begin{array}{l}\text { oposición. } \\
\text { - 3a. Acciones de } \\
\text { cooperación } \\
\text { - 3b. Acciones de } \\
\text { cooperación y oposición. }\end{array}$ \\
\hline $\begin{array}{l}\text { 5-Acciones en entorno físico con incertidumbre. Actividades } \\
\text { en un medio desconocido o, fundamentalmente, en el medio } \\
\text { natural. La clave es la correcta interpretación de las variaciones } \\
\text { en el medio. Tiene gran importancia la seguridad. }\end{array}$ & $\begin{array}{l}\text {-4. Acciones motrices en } \\
\text { situaciones de adaptación } \\
\text { al entorno físico }\end{array}$ \\
\hline $\begin{array}{l}\text { 6-Acciones con intenciones artísticas y/o expresivas. } \\
\text { Acciones con finalidades estéticas y comunicativas. También } \\
\text { suele haber una relación estrecha con el ritmo. Se prestan } \\
\text { mucho a la organización de proyectos de acción colectiva. }\end{array}$ & $\begin{array}{l}\text { - 5. Acciones motrices en } \\
\text { situaciones de índole } \\
\text { artística o de expresión. }\end{array}$ \\
\hline \multicolumn{2}{|c|}{$\begin{array}{l}\text { (**Nota aclarativa: la propuesta original de Larraz (2004) del DAM } 4 \text { es: "Acciones de } \\
\text { cooperación-oposición". Velázquez (2012) sostiene que se trata de un uso incorrecto del } \\
\text { término "cooperación", dado que para que sea realmente cooperación no es posible la } \\
\text { oposición, el enfrentamiento ni la competición. Por ello, éste autor sostiene que la } \\
\text { denominación correcta debería ser "colaboración-oposición". Por esta razón hemos } \\
\text { cambiado la denominación original de Larraz). }\end{array}$} \\
\hline
\end{tabular}

\section{Ventajas de programar por Dominios de Acción Motriz en Edu-} cación Física

Entendemos que el punto más fuerte de esta propuesta es que su aplicación al mundo de la EF puede ser interesante a la hora de dotar nuestra área de un mayor rigory estructura. Realizar la programación de EF por DAM permite realizar un proceso de aprendizaje con mucha más lógica, sistematicidad, rigor y cercanía a la realidad cotidiana del alumnado. Lo mismo puede aplicarse al encadenamiento de sesiones dentro de una unidad didáctica y a las actividades concretas de aprendizaje a desarrollar en el aula. Este planteamiento puede ser de una gran ayuda a la hora de ir clarificando qué se quiere enseñar y cuándo y cómo hacerlo; y, sobre todo, a la hora de organizar mejor el proceso de enseñanza-aprendizaje en EF, al trabajar sobre la lógica interna de las diversas situaciones motrices. Larraz (2009) considera que trabaja por DAM en EF tiene las siguientes ventajas: (1) permite organizar las prácticas de la EF; (2) facilita la programación didáctica de la EF; (3) posibilita organizar los contenidos de los currículos de EF; (4) facilita el aprender a ser personas y (5) determina las competencias específicas de la EF. Y 
lo resume en una idea principal: dan coherencia interna a la EF.

Desde nuestro punto de vista, tiene también otras ventajas complementarias: (1) permite programa de forma equilibrada y coherente cada curso y durante los seis cursos de la etapa; (2) ayuda al profesorado a tener claro qué queremos que aprenda nuestro alumnado y cómo organizar su aprendizaje; (3) permite secuenciar por ciclos y etapas con criterios mucho más lógicos y equilibrados; (4) ayuda a secuenciar las tareas y actividades con criterios lógicos de aprendizaje; (5) permite que todo el alumnado pueda tener éxito en EF; (6) posibilita trabajar con «actividades físicas de referencia» que permiten conectar la EF con la vida real y complementarse mutuamente; (7) proporciona al alumnado un bagaje motor lo más diverso, equilibrado y completo posible tras su paso por los seis cursos de primaria.

Como punto débil, habría que señalar que se trata de un planteamiento poco conocido actualmente entre el profesorado de EF y del que se han publicado pocas propuestas prácticas concretas aunque, afortunadamente, poco a poco va incrementándose su número (Abós, GarcíaGonzález, Sevil y Sanz, 2015; Barba y López, 2006; Generelo, Julián y Zaragoza, 2009; Generelo, Zaragoza y Julián, 2005; Julián, Aguareles, Ibor, Cervantes y Abarca, 2013; Julián, Generelo, García, Abarca-Sos y Zaragoza, 2012; Julián y Pinos, 2011; Julián y Zaragoza, 2014; Julián, Zaragoza, López y Generelo, 2005; Julián, Zaragoza, López y Generelo, 2007; López-Pastor et al., 2003; López-Pastor, Monjas y PérezBrunicardi, 2003; López-Pastor y Hernández, 2015; Manrique, Vacas y Gonzalo, 2011; Monjas, 2006; Ponce, López y Jiménez, 2015). Puede encontrarse un buen número de interesantes propuestas prácticas basadas en los DAM en el blog «Educación Física Escolar», de Alfredo Larraz: http://www.educacionfisicaescolar.es/, así como en la web del grupo de investigación «Educación Física y Promoción de la Actividad Física» (EFYPAF): http://efypaf.unizar.es/recursos/ dominiosAF.html\#organizaciondominios.

\section{Los futuros de la Educación Física}

En un interesante trabajo sobre los retos de futuro de la EF actual, Kirk (2008) analiza la evolución que ha sufrido la «idea de la idea de EF» a lo largo de su presencia en el sistema educativo, para intentar realizar una previsión de cuál puede ser el escenario en un futuro próximo. Una de las conclusiones que plantea es que podríamos estar a las puertas de un nuevo cambio, en la dirección del plan de actuación para la EF y el deporte aprobado en Gran Bretaña en 2008 ( «Physical Education and Sport Strategy for Young People), que establece tres tipos de programas de EF y Deportes: (a) el orientado al rendimiento deportivo; (b) el orientado a la lucha contra la obesidad; y (c) el orientado al desarrollo de conductas prosociales.

En oposición a este futurible, Kirk (2008) considera que el desafío de cualquier materia escolar es triple: (1) la transferencia del aprendizaje escolar a la vida durante y después de la escuela; (2) la autenticidad del conocimiento escolar en relación a la vida; y (3) el papel de la escuela como institución de transformación social. Esta idea también podemos aplicarla a la EF. Si lo planteamos en forma de preguntas serían las siguientes: (a) ¿nuestro alumnado puede trasferir los aprendizajes que adquiere en EF a su vida cotidiana, durante y después del tiempo escolar?; (b) ¿los aprendizajes y conocimientos que generamos en EF actualmente son auténticos, tienen conexión con la vida real?; y (c) ¿la EF aporta algo actualmente a la función de trasformación social que tiene la escuela?

Teniendo en cuenta estas preguntas, entendemos que la mayoría de las propuestas y corrientes revisadas en el punto anterior realizan aportaciones en mayor o menor grado a este desafío. Esto es, la mayoría de estas propuestas de EF generan aprendizajes relevantes para la vida del alumnado y, en algunos casos, también tienen una clara finalidad de transformación social. física

Las competencias profesionales del profesorado de educación

Desde nuestro punto de vista, cuando se habla del tema de competencias profesionales lo esencial es tener una serie de ideas básicas bien definidas, para luego actuar en consecuencia. Aquí vamos a intentar recopilar las que han ido conformando nuestra forma de entender esta profesión y la formación del profesorado:

1-Una competencia implica la aplicación integrada de cuatro capacidades básica: saber, saber hacer, saber ser y saber estar. En el informe Delors (1996) sobre el tesoro que encierra la educación puede encontrarse un interesante desarrollo de estos aspectos.

2-Una competencia implica la aplicabilidad de las capacidades en situaciones reales. Por tanto, es un paso importante más allá del simple y puro conocimiento. Los conceptos de aprendizaje auténtico y evaluación auténtica pueden ser muy útiles para entender esto y, sobre todo, para avanzar en propuestas prácticas en este sentido (López-Pastor, 2013).

3-Avanzar hacia modelos de formación inicial o permanente basados en competencias supone la posibilidad de avanzar hacia modelos de aprendizajemás integrales y hacia el desarrollo de capacidades cognitivas complejas.

4-Consideramos fundamental que la formación inicial del profesorado de EF tenga un enfoque mucho más competencial y práctico de lo que ha tenido en las últimas décadas.

Para poder establecer con claridad y prioridad cuáles son las competencias profesionales que debería tener el profesorado de EF, primero deberíamos tener muy claro cuáles son los fines de la EF para los que deben ser formados. En ese sentido, y tras lo explicado en los apartados anteriores, el profesorado de EF debería desarrollar competencias sobre los siguientes aspectos: (1) el desarrollo físico-motriz del alumnado; y (2) el desarrollo de procesos de aprendizaje en las diferentes prácticas sociales de referencia de cada uno de los seis DAM. Esto implicaría el desarrollo de, al menos, seis asignaturas diferentes, una por cada DAM, en las que trabajar varios aspectos: (a) la vivencia personal de «prácticas de éxito»; (b) el dominio de las competencias fundamentales de cada DAM y su aplicación en situaciones educativas; (3) el diseño y aplicación tutorizada de propuestas concretas; y (4) su aportación al planteamiento global de desarrollo integral del alumnado, como ciudadanos de una sociedad democrática. Hay experiencias en esta línea, como el plan de estudios del grado de Magisterio en Educación Primaria de la Universidad de Zaragoza (Enlace: http://titulaciones.unizar.es/maestro-ed-primaria/cuadro_asignaturas.html).

En este sentido, el proceso de Convergencia hacia el Espacio Europeo de Educación Superior(EEES) parecía una buena oportunidad para avanzar en esta dirección. Esta perspectiva positiva, no obstante, conlleva la duda de si realmente la universidad va a permitir avanzar en la dirección adecuada, ya que existen en muchas ocasiones obstáculos y hábitos inadecuados a nivel de organización y funcionamiento. Nos estamos refiriendo a situaciones como, por ejemplo: (a) la escasa importancia y tiempo dedicado por el profesorado universitario a mejora la calidad de la docencia frente al impacto que tiene la investigación sobre la promoción profesional; (b) la fuerte tendencia de la universidad hacía al inmovilismo didáctico y la reproducción del aprendizaje bancario (dictado de apuntes-diapositivas y examen final) sin avanzar hacia metodologías inductivas; (c) la desorganización interna en el reparto y organización de la docencia por intereses personales sin atender los de los estudiantes; (d) la aparente imposibilidad de coordinarse en la docencia de asignaturas compartidas; y (e) el plantear los nuevos planes de estudio con la obsesión por el «reparto de la tarta» entre departamentos, etc.

A partir de estas consideraciones, planteamos dos hipótesis para un futuro cercano en cuanto a las competencias profesionales que las nuevas generaciones de docentes deben adquirir para el correcto progreso de la EF a lo largo de los próximos años:

(a)-Respecto al profesorado de primaria (Grado Magisterio Primaria, mención Educación Física). Estamos convencidos de que su formación como generalistas va a ser considerablemente mejor, en términos generales, que la que tuvieron las generaciones que estudiaron la antigua diplomatura. Hay tres razones básicas para afirmar esto: hay un curso más de formación inicial, hay uno o dos periodos más de prácticas y hay más formación específica como generalistas a lo largo de los diferentes 
cursos del Grado. En cambio, su formación especializada en EF nos plantea más dudas. Por un lado, siguen existiendo unas diferencias excesivamente grandes en función de la universidad en la que se estudie, desde los 24 créditos específicos de alguna universidad española hasta los 72 de otras. Por otro, la formación específica con la que salen los actuales graduados «especialistas» en EF es claramente insuficiente y, en muchas facultades, bastante inferior a la que de daba a los antiguos diplomados de magisterio en $\mathrm{EF}$.

(b)-Respecto al profesorado de secundaria, graduados en Ciencias de la Actividad Física y el Deporte más el Máster de Profesorado de Secundaria, la situación es diferente. Por un lado la evolución de los planes de estudio en los últimos 15 años ha supuesto la práctica desaparición de la «Educación Física» en la titulación, frente a corrientes más deportivas, biomédicas y psicológicas. Por otro, la implantación del Máster específico para ejercer docencia en Secundaria supone una considerable evolución positiva respecto al anterior y caótico curso de adaptación pedagógica (CAP). En teoría, debería suponer una mejora importante en la formación de competencias profesionales específicas para tan complicada etapa educativa. Pero la triste realidad de la universidad española es que sigue siendo difícil asegurar que este cambio y mejora aproveche todo su potencial en todas las universidades, dado que en muchos casos puede suponer, una vez más, la socialización profesional en metodologías tradicionales, alejadas de la normativa educativa actual y que poco ayudan a romper los círculos viciosos en que llevamos moviéndonos en las últimas décadas.

Pero también hay motivos para la esperanza. Como hemos visto en apartados anteriores, durante las tres últimas décadas han surgido en España muchas propuestas innovadoras en EF que pueden ser muy útiles para ir dando una respuesta educativamente valiosa a estos retos. La mayor parte de dichas propuestas han sido generadas por el profesorado de EF a través de dinámicas de investigación-acción e investigación en el aula. Por otra parte, la Universidad de Elche está organizando, desde el 2014, un interesante Simposium sobre «Enseñanza físicodeportiva fundamentada en evidencias»; y como bien explica Flecha (2015), muchas prácticas educativas siguen estando basadas en ocurrencias, en vez de en evidencias, en tradiciones sin fundamento, en prejuicios y charlas de cafetería, en vez de en un conocimiento profesional sólido y fundamentado. Cada vez más hay compañeros que están convencidos de que la práctica educativa debería estar sólidamente basada en los resultados de la investigación educativa, en evidencias demostrables de cuáles son las mejores prácticas educativas, las «prácticas de éxito» que generan los mejores resultados educativos en todo el alumnado (Aubert, Flecha, García, Flecha y Racionero, 2008; Flecha y Alvarez, 2015). La formación inicial y permanente del profesorado tiene un gran margen de mejora si comienza a basarse en este tipo de planteamientos.

Conclusiones. Cerrando el círculo: ¿Cuál podría ser el principal reto de la EF y del profesorado de EF?

A modo de conclusión vamos a plantear dos conclusiones:

(a)-Sólo cuando tengamos claros cuáles son los fines de la EF podremos comenzar a establecer con claridad y prioridad cuáles son los retos actuales de la EF y cuáles son las competencias profesionales que debería tener el profesorado de EF.

(b)-Nuestro principal reto en estos momentos sigue siendo el desarrollo de una práctica educativa de calidad, fundamentada y coherente. Es complicado que nuestro estatus mejore mientras sigamos dando bandazos al ritmo de la moda o desarrollando prácticas incoherentes con los principios educativos más básicos y fundamentales.

Por supuesto, son tan sólo unas conclusiones abiertas a la discusión, el debate y la comprobación práctica. Tanto el debate como la crítica argumentada y positiva serán bienvenidas.

\section{Agradecimientos}

A lo largo de los últimos veinte años hemos compartido trabajo, diálogos, interés y aprendizaje con muchas compañeras y compañeros de profesión en jornadas, congresos, cursos, encuentros y talleres. Hablando con ellas y con ellos se ha ido construyendo nuestra forma de entender y practicar esta profesión tan apasionante que compartimos. Así que muchas gracias por lo que os corresponde en ese proceso de crecimiento profesional y personal. Esperamos poder seguir con él muchos años más.

\section{Referencias}

Abós,A., García-González,L., Sevil, J. y Sanz, M. (2015). «Variables motivacionales influyentes en una unidad didáctica de rugby: claves para la mejora de la intervención docente». Scientific Technical Journal of School Sport, Physical Education and Psychomotricity, 1(6), 106-122.

Alonso, M. C., Gómez-Alonso, M. T., Pérez-Pueyo, A. y Gutiérrez-García, C. (2016). «Errores en la intervención didáctica de profesores de educación físicaen formación en sesiones simuladas desde la perspectiva de sus compañeros». Retos de la Actividad Física y el Deporte, 29, 229-235.

Aubert, A., Flecha, A., García, C., Flecha, R. y Racionero, S. (2008). Aprendizaje dialógico en la Sociedad de la Información. Barcelona: Hipatia Editorial.

Barba, J. J.(2006). Aprendiendo a ser maestro en una escuela unitaria. Vivencias, sensaciones y reflexiones en la primera oportunidad. Morón(Sevilla): MCEP.

Barba, J. J. y López, V. M. (coords) (2006). Aprendiendo a correr con autonomía: buscando un ritmo constante y sostenible en esfuerzos de larga duración. Unidades Didácticas y Experiencias en Educación Primaria, Secundaria Obligatoria y Bachillerato. Buenos Aires: Miño y Dávila.

Castejón, J. (2015). «La investigación en iniciación deportiva válida para el profesorado de educación física en ejercicio». Retos de la Actividad Física y el Deporte, 28, 263-269.

Córdoba, C. (2015). La aventura de aprender: Relato autobiográfico del viaje a Ítaca de un docentereflexivo. Retos de la Actividad Física yel Deporte,28, 285-290.

Córdoba, C.; Carbonero, L.; Sánchez, D.; Inglada, S.; Serra, M.; Blasco, M.; Sáez, S.; Ivanco, P. (2016). «Educación Física Cooperativa, formación permanentey desarrollo profesional. Dela escritura colectiva a un relato de vida compartido». Retos de la Actividad Física y el Deporte, 29, 264-269.

Delgado, M. A. (2015). «Los estilos de enseñanza de la Educación Física y el Deporte a través de 40 años de vida profesional». Retos de la Actividad Física yel Deporte, 28,240-247.

Delors, J. (coord.) (1996). La educación encierra un tesoro. UNESCO.

Devís, J. y Peiró, C. (1993). «La actividad física y la promoción de la salud en niños/ as y jóvenes: la escuela y la educación física». Revista de Psicología del deporte, 4, 71-86.

EFYPAF (2012) Grupo de investigación Educación Física y promoción de la actividad física. Recuperado: http://efypaf.unizar.es/recursos/ dominiosAF.html\#organizaciondominios Consultado el 22-09-2015).

Fernández-Rio, F. J.y Méndez,A. (2016). «El Aprendizaje Cooperativo: Modelo Pedagógico para Educación Física». Retos de la Actividad Física yel Deporte, 29, 201-206.

Flecha, R. (2015). «Comunidades de aprendizaje: sueños posibles para todas las niñas y los niños». Aula de innovación educativa, 241,12-16.

Flecha, R. y Alvarez, P. (2015). «Investigación educativa e impacto social: Claves para mejorar la educación de todos los niños y niñas». Revista Padres y Maestros / Journal of Parents and Teachers, 362,15-19.

Flores, G, Prat, M. y Soler, S. (2015). «La intervención pedagógica del profesorado de educación física en un contexto multicultural: prácticas, reflexiones y orientaciones». Retos de la Actividad Física y el Deporte, 28,248-255.

Gallardo, F. J. \& Carten, B. (2016). La evaluación formativa y compartida en la formación inicial del profesorado. Análisis de un caso en Chile. Retos de la Actividad Física y el Deporte, 29, 258-263

Generelo, E., Julián, J. A. y Zaragoza, J. (2009). Tres vueltas al patio. La carrera de larga duración en la escuela. Barcelona. INDE.

Generelo, E., Zaragoza, J. y Julián, J.A. (2005): La Educación Física en las aulas: Aprender a partir de un proyecto. Zaragoza. Departamento de Educación, Cultura y Deporte.

González-Calvo, G; Barba, J. J. (2013). «Perspectiva autobiográfica de un docente novel sobre los aprendizajes de Educación Física en diferentes niveles educativos en educación física». Cultura, Ciencia y Deporte, 24, 171-181.

González,F. J. (2016). El nuevo currículum de educación física en Brasil: un desafío de futuro. Retos de la Actividad Física y el Deporte, 29, 188-194.

Julián Clemente, J.A. Generelo Lanaspa, E., García González, L.,Abarca-Sos, A.y Zaragoza Casterad (2012). «Estrategias para fomentar un clima motivacional óptimo en el contenido de carrera de larga duración en la Educación Física escolar». Revista Tándem. Didáctica de la Educación Física, 40, 54-65.

Julián, J.A., Abarca-Sos, A, Zaragoza, J. \& Aibar, A. (2016). Análisis crítico de la propuesta del currículum básico dela LOMCE para la asignatura de Educación 
Física.Acciones derivadas en la Comunidad Autónoma deAragóny propuestas de futuro. Retos de la Actividad Física y el Deporte, 29, 173-181

Julián, J.A. y Pinos, M. (2011). Ejemplificación de educación física para segundo ciclo de primaria. Actividad: Orientación. Zaragoza. Departamento de Educación, Universidad, Cultura y Deporte del Gobierno deAragón. Recuperado de: http://efypaf.unizar.es/recursos/orientacion.html

Julián, J. A. y Zaragoza, J. (2014). Unidad didáctica de las actividades de bate y carrera (golpeo y fildeo) para segundo ciclo de primaria. En Méndez-Giménez, A.(Coord). Modelos de enseñanza en educación física: unidades didácticas de juegos deportivos de diana móvil, golpeo y fildeo y pared. Editorial Grupo 5, Madrid, 151-184.

Julián, J. A., Aguareles, I., Ibor,E., Cervantes, J. y Abarca, A. (2013). El esquí nórdico en la escuela. Propuesta de aprendizaje para el marco escolar en Educación primaria. Habilidad motriz: Revista de ciencias de la actividad fisicay del deporte, 41, 44-55.

Julián, J.A., Zaragoza, J., López, N. y Generelo, E. (2005). El trabajo de combas en la ESO a través de la construcción de un proyecto de acción. TANDEM. Didáctica de la Educación Física, 19, 79-91.

Julián, J. A., Zaragoza, J., López, N. y Generelo, E. (2007). «¿Bailar en el agua? Estructura de una unidad didáctica de natación sincronizada mediante un proyecto de acción». TANDEM. Didáctica de la Educación Física, 23, 98-112.

Kirk, D. (2008). Los futuros de la Educación Física: la importancia de la cultura física y de la «idea de la idea» de Educación Física. En AA. VV. Actas V Congreso Asociación Española de Ciencias del Deporte. León: Universidad de León. (CD-R).

Larraz, A. (2002). Diseños curriculares de la comunidad autónoma de Aragón Educación Primaria: Educación Física. (consultado 5-5-2008). Recuperado de:(http://www.praxiologiamotriz.inefc.es/PDF/Praxio_Lleida_curi_011.pdf).

Larraz, A. (2004). Los dominios de acción motriz como base de los diseños curriculares en educación física: el caso de la Comunidad deAragón en educación primaria. En Lagardera, F. y Lavega, P. (Ed.) La ciencia de la acción motriz. Lleida: Universitat de Lleida, 203-226.

Larraz,A. (2009).¿Quéaprendizajes de educación física debería tener el alumnado al finalizar sexto curso de primaria?. Tándem. Didáctica de la Educación Física, $29,45-63$.

Larraz, A. (2014). Educación Física Escolar. El blog de Alfredo Larraz Urgelés. Recuperadode:http://www.educacionfisicaescolar.es/ (consultadoel24-09-2015).

López Pastor, V. M. et al. (2004). «Las Historias de vida en la formación inicial de profesorado de Educación Física». Revista Internacional de Medicina y Ciencias de la Actividad Física y el Deporte, 4(13). Recuperado de: http:// cdeporte.rediris.es/revista/revista13/evaluavida.html

López Pastor, V. M. et al. (2005). ¿Cuando Cuándo tenemos poco prestigio? Mirando la viga en el ojo propio. Retos de Educación Física y deportes, 8, 11 18.

López-Pastor, V. M. (2013). Evaluación en Educación Física. Revisión internacional de la temática. Revista de Educación Física. Renovar la teoría y la práctica, 29(3), 4-13.

López-Pastor, V. M. et al. (2003). Unidad didáctica interdisciplinar de iniciación a teatro de sombras y multiculturalidad en Educación Física. Revista La Peonza 3, 18-24.

López-Pastor, V. M., Ruano, C., Hernangómez, A., Cabello,A., Hernández, B. et al.(2016). Veinteaños de formaciónpermanente del profesorado, investigaciónacción y programación por dominios de acción. Retos de la Actividad Física y el Deporte, 29, 270-279.

López-Pastor, V. M. y Hernández Sánchez, B. (2015). Iniciación al teatro de luz negra. Una experiencia diferente de expresión corporal. En: XI Congreso Internacional sobre la enseñanza de la educación física y el deporte escolar (capítulo 19), [DVD]. Alcoy: Alto Rendimiento.

López-Pastor, V. M., et al. (2003). Cuestionando algunos tópicos en Educación Física: la estructura de sesión y los juegos como recurso didáctico. Revista La Peonza, 4, 6-9.

López-Pastor, V. M., Monjas, R. y Pérez-Brunicardi, D. (2003). Buscando alternativas a la forma de entender y practicar la educación física escolar. Barcelona: Inde.

López-Pastor, V.M. y Gea, J. M. (2010). Innovación, discurso y racionalidad en Educación Física. Revisión y prospectiva. Revista Internacional de Medicina y Ciencias de la Actividad Física y el Deporte, 10(38), 245-270. Recuperado de:Http://cdeporte.rediris.es/revista/revista38/artinnovacion154.htm.

Manrique, J. C., Vacas, R. y Gonzalo, A. (coords.) (2011). Las habilidades físicas básicas: una buena oportunidad para la cooperación. Unidades didácticas y experiencias en educación primaria. Buenos Aires: Miño y Dávila.

Martínez,L. (2016). Autoevaluación de competencias profesionales y proyectos de aprendizajetutorados en formacióninicial del profesorado. Retos de la Actividad Física y el Deporte,29, 242-250.

Martos, D.; Tamarit, E.y Torrent, G. (2016). Negociando el currículum en educación física. Una propuesta práctica de cogestión. Retos de la Actividad Física y el Deporte, 29. 223-228.
Monjas Aguado, R. (coord.) (2006). La iniciación deportiva en la escuela desde un enfoque comprensivo. Experiencias y materiales curriculares. Buenos Aires: Miño y Dávila.

Monjas, R. Ponce, A. y Gea, J. M. (2015). La trasmisión de valores a través del Deporte. Deporte Escolary Deporte Federado: relaciones, puentes y posibles trasferencias. Retos de la Actividad Física y el Deporte, 28,275-284.

Monzonís, N. y Campllonch, M. (2015). Mejorar la competencia social y ciudadana: innovación desde Educación Física y tutoría. Retos de la Actividad Física yel Deporte, 28, 256-262.

Moreno, A.; Poblete, C. (2015). La Educación Física Chilena y su profesorado: proponiendo algunos retos para la investigación en el área. Retos de la Actividad Física y el Deporte, 28,291-196.

Orden EDU/519/2014, de 17 de junio (2014), por la que se establece el currículoy se regula la implantación, evaluación y desarrollo de la educación primaria en la Comunidad de Castilla y León (BOCYL, 117).

Pastor-Vicedo, J.C., Gil-Madrona, P., Prieto-Ayuso, A. y González-Vilora, S. (2015). Los contenidos de salud en el área de educación física: Análisis del currículum vigente. Retos de la Actividad Física y el Deporte, 28, 134-140.

Pedraza, M. A. y López-Pastor, V. M. (2015). «Investigación-acción, desarrollo profesional del profesorado de educación física y escuela rural». Revista Internacional de Medicina y Ciencias de la Actividad Física y del Deporte, 57,1-16.

Pérez-Brunicardi, D. (2002). «Envidia Sana». Cuadernos Pastopas. Revista de intercambio de experiencias sobre lo corporal y lo motriz, 1, 4.

Pérez-Pueyo,A. (2016). El Estilo Actitudinal en Educación Física: Evolución en los últimos 20 años. Retos de la Actividad Física y el Deporte,29, 207-215.

Ponce, A., Lopez-Pastor, V. M. y Jiménez, B. (2015). «Dramatización de canciones». En: XI Congreso Internacional sobre la enseñanza de la educación física yel deporte escolar (capítulo 20). Alcoy:Alto Rendimiento. (DVD).

Programa Perseo (2007). Actividad física saludable. Guía para el profesorado de Educación Física. Madrid: Ministerio de Sanidad y Consumo y Ministerio de Educación y Ciencia.

Real Decreto 126/2014, de 28 de febrero, por el que se establece el currículo básico delaEducación Primaria(BOE, nº 52 de 1 demarzo de 2014; 19.349-19.420).

Romero, R., Asún, S. y Chivite, M. (2016). La autoevaluación en expresión corporal en formación inicial del profesorado de educación física como ejemplo de buena práctica. Retos de la Actividad Física y el Deporte,29, xx-Xx

Ruiz-Omeñaca, J. V., Ponce, A., Sanz, E. y Valdemoros, M. A. (2015). «La educación en valores desde el deporte: investigación sobre la aplicación de un programa integral en deportes de equipo». Retos de la Actividad Física y el Deporte, 28,270-275.

Tabernero, B. (2016). ¿ Jugamos a los cuentos? Una propuesta práctica de animación a la lectura a través de la Educación Física. Retos de la Actividad Física y el Deporte, 29. 216-222.

Tinning, R. (1996). Discursos que orientan el campo del movimiento humano y el problema de la formación del profesorado. Revista de Educación, 311, 123-134.

Velázquez, C. (2015). «Aprendizaje Cooperativo en Educación Física: estado de la cuestión y propuesta de intervención». Retos de la Actividad Física y el Deporte,28, 234-239.

Velázquez, C. (coord.) (2012). Aprendizaje cooperativo en Educación Física. Fundamentos y aplicaciones prácticas. Barcelona: Inde.

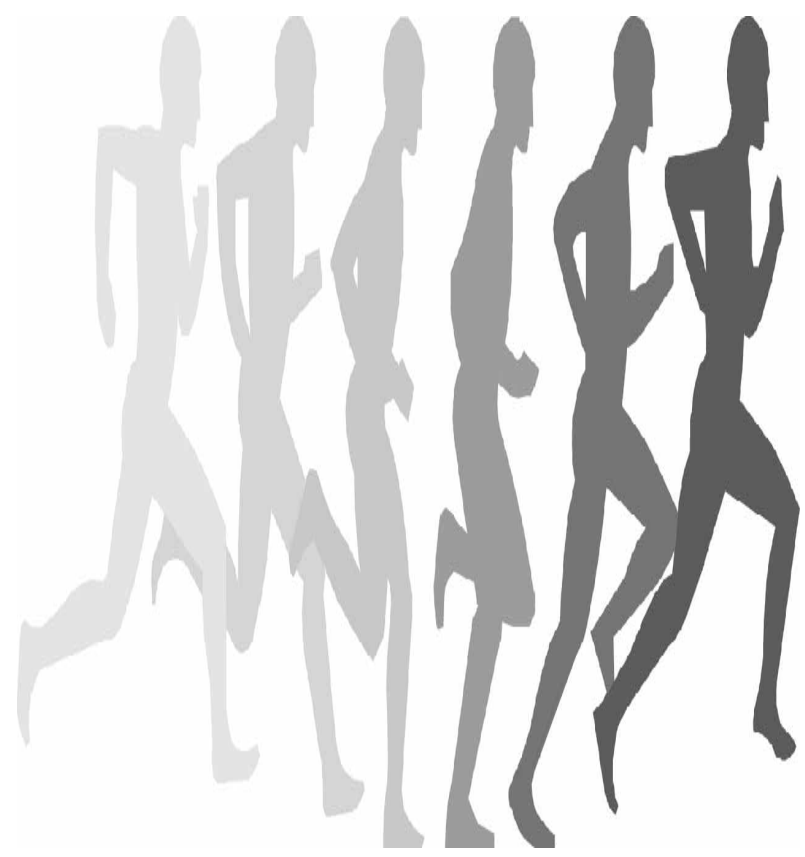

